\title{
Líquen plano: a história de uma cooperação de sucesso
}

Sofia Sousa e Silva, ${ }^{1}$ Catarina Meireles, ${ }^{1}$ Fátima Costa, ${ }^{2}$ Susana Carvalho ${ }^{3}$

\section{RESUMO}

Introdução: A patologia dermatológica é muitas vezes motivo de consulta em medicina geral e familiar. O caso clínico descrito corresponde ao diagnóstico de líquen plano, que é um problema de saúde pouco frequente. O líquen plano é uma doença inflamatória crónica, imuno-mediada e de etiologia desconhecida que afeta entre 0,22 a 5\% da população mundial.

Descrição do caso: Sexo masculino, 60 anos de idade, sem antecedentes patológicos de relevo. Em outubro de 2014 recorre à consulta de cuidados de saúde primários por apresentar quadro com cerca de um mês de evolução, caracterizado por pápulas pruriginosas nas mãos, tronco, dorso e pés. Apresentava ainda estriação das unhas das mãos com dor à palpação do leito ungueal. Ao exame objetivo apresentava pápulas poligonais, achatadas e bem definidas, de coloração violácea, agrupadas e confluentes, na face anterior dos punhos, na região anterior do tórax, no dorso e na face anterior dos tornozelos. Como lesões associadas apresentava hipocratismo digital, eritema palmar, eritema periungueal, com atingimento da matriz, adelgaçamento da lâmina ungueal, sulcos longitudinais em toda a superfície ungueal e dor à palpação da raiz das unhas. Foi pedida colaboração de dermatologia via ALERT ${ }^{\circledR}$, tendo sido sugerido o diagnóstico de líquen plano. Iniciou terapêutica com corticoterapia tópica que não foi eficaz, tendo posteriormente iniciado griseofulvina 500mg/dia, com melhoria do quadro.

Comentário: Este caso clínico enfatiza a importância da comunicação entre especialidades para a abordagem dos problemas do doente, de forma a agilizar o tratamento e a melhorar a qualidade de vida.

Palavras-chave: Líquen plano; Griseofulvina.

\section{INTRODUÇÃO}

A patologia dermatológica é muitas vezes motivo de consulta em medicina geral e familiar. Em 2010, um estudo publicado na Revista Portuguesa de Medicina Geral e Familiar revelou que a pele foi motivo de consulta em $2,8 \%$ dos utentes. ${ }^{1}$

A grande variedade de patologia relacionada com o foro dermatológico e a baixa prevalência da maioria das doenças dermatológicas é um grande desafio à capacidade de diagnóstico e de gestão de problemas do médico de família.

O caso clínico descrito corresponde ao diagnóstico de líquen plano, que é um problema de saúde pouco

'Médicas Internas de Medicina Geral e Familiar. USF Serpa Pinto, ACeS Porto Ocidental, ARS Norte

${ }^{2}$ Médica Assistente de Medicina Geral e Familiar. USF Citânia, ACeS Vale do Sousa Norte, ARS Norte

${ }^{3}$ Médica Assistente de Medicina Geral e Familiar. USF Serpa Pinto, ACeS Porto Ocidental, ARS Norte frequente. O líquen plano é uma doença inflamatória crónica, imuno-mediada e de etiologia desconhecida que afeta entre 0,2 a $5 \%$ da população mundial. ${ }^{2}$

Este caso clínico enfatiza a importância da comunicação entre especialidades para a abordagem dos problemas do doente, de forma a agilizar o tratamento e a melhorar a qualidade de vida.

\section{DESCRIÇÃO DO CASO}

Descreve-se o caso clínico de um homem de raça caucasiana, 60 anos de idade, sem antecedentes pessoais de relevo. Como antecedentes familiares, apresenta pai com doença de Alzheimer.

Inserido numa família nuclear, composta pelo próprio e pela companheira. Divorciado, dois filhos de uma relação anterior, de 34 e 37 anos. Esta família pertence à classe III de Grafar.

Hábitos de vida: ex-fumador desde há dois anos (88UMA), consumo de 252g por semana de álcool, sem cuidados alimentares, estilo de vida sedentário. 

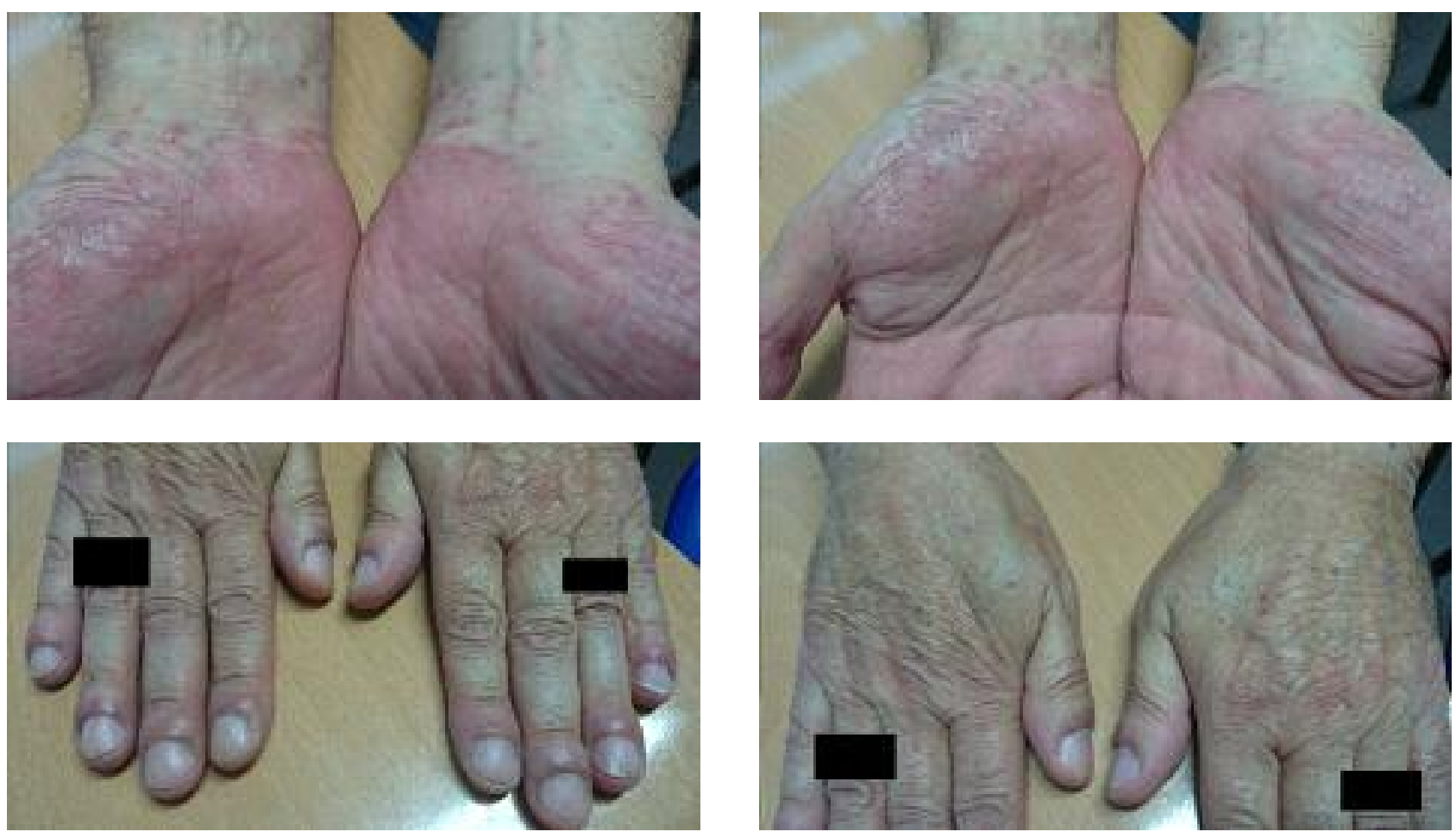

Ilustração 1 a 4. Lesões cutâneas e ungueais de líquen plano do utente (autorizadas pelo mesmo).

Em outubro de 2014 recorre pela primeira vez à consulta de medicina geral e familiar por apresentar quadro com cerca de um mês de evolução caracterizado por pápulas pruriginosas nas mãos, tronco, dorso e pés. Apresentava ainda estriação das unhas das mãos com dor à palpação do leito ungueal. Em agosto de 2014 tinha recorrido ao urologista privado por lesões vesiculares não dolorosas na glande, tendo sido diagnosticado com candidíase e realizado tratamento antifúngico tópico com resolução da lesão.

Ao exame objetivo apresentava pápulas poligonais, achatadas e bem definidas, de coloração violácea, agrupadas e confluentes, na face anterior dos punhos, na face anterior do tórax, no dorso e na face anterior dos tornozelos. Como lesões associadas apresentava hipocratismo digital, eritema palmar, eritema periungueal, com atingimento da matriz, adelgaçamento da lâmina ungueal, sulcos longitudinais em toda a superfície ungueal e dor à palpação da raíz das unhas.

Foi requisitado estudo analítico, cujos resultados in- dicavam dislipidemia, sem outras alterações. Os marcadores víricos da hepatite B e C e VIH 1 e 2 eram negativos, assim como o VDRL era não reativo.

Perante as lesões encontradas foi decidido referenciar para dermatologia, tendo sido enviadas as fotos do doente via ALERT ${ }^{\circledR}$. O colega de dermatologia enviou resposta pela mesma via, sugerindo o diagnóstico de líquen plano. Propôs início do tratamento com corticoterapia tópica de elevada potência durante cerca de seis meses. Como tratamento alternativo sugeriu o uso off-label de griseofulvina $500 \mathrm{mg} /$ dia durante seis meses.

Em novembro de 2014, o paciente iniciou tratamento com butirato de hidrocortisona $1 \mathrm{mg} / \mathrm{g}$ na formulação de creme, na posologia de duas aplicações diárias nas lesões.

Em dezembro de 2014, o paciente recorre novamente à consulta, com queixas de agravamento das lesões dos punhos e das lesões ungueais, com aumento da dor na raiz das unhas das mãos. Nesta consulta é explicado ao doente a possibilidade de realizar tratamento com gri- 
seofulvina off-label, por indicação do colega de dermatologia, que teria que ser comprada no estrangeiro por não ser comercializada em Portugal. É agendada consulta de dermatologia para setembro de 2015, nove meses após a referenciação inicial.

Em março de 2015, o doente recorre novamente à consulta. Está a cumprir o esquema terapêutico com griseofulvina 500mg/dia desde janeiro de 2015, que tem comprado em França. Apresenta melhoria franca das lesões cutâneas e das lesões ungueais. Neste momento propõe-se ao doente manter a terapêutica até junho de 2015, aguardando consulta de dermatologia.

\section{COMENTÁRIO}

O líquen plano é uma doença inflamatória crónica imuno-mediada de etiologia desconhecida. O seu início é geralmente agudo e pode afetar a pele, as unhas, o cabelo e as mucosas. ${ }^{3}$ Envolve preferencialmente as superfícies flexoras das extremidades e apresenta-se como pápulas violáceas pruriginosas. Habitualmente utiliza-se a mnemónica dos $6 P$-pápulas, placas, planas, poligonais, pruriginosas e púrpuras. ${ }^{2}$ Estas lesões podem estar cobertas por linhas reticulares e brancas, conhecidas por estrias de Wickham. Após a resolução das lesões cutâneas é frequente existir hiperpigmentação na zona afetada. ${ }^{3-4}$

Estima-se que a prevalência mundial esteja compreendida entre os $0,2 \%$ e os $5 \% .{ }^{2}$ Pode aparecer em qualquer idade, mas na maioria dos casos verifica-se entre os 30 e os 60 anos. $^{3}$

Existem vários subtipos de líquen plano. A divisão é baseada na localização cutânea $v s$ a localização nas mucosas. As formas cutâneas de líquen plano subdividem-se em: líquen plano linear; líquen plano anelar; líquen plano atrófico; líquen plano hipertrófico; líquen plano vesiculo bolhoso; líquen plano erosivo/ulcerativo. ${ }^{3}$ As formas mucosas podem abranger a mucosa oral, a mucosa genital e esofágica. ${ }^{2}$

Cerca de $10 \%$ dos doentes apresentam lesões no couro cabeludo e nas unhas. As lesões do couro cabeludo são pápulas pruriginosas e violáceas que podem progredir para alopécia se não forem tratadas. $\mathrm{O}$ envolvimento das unhas caracteriza-se por ondulação longitudinal da placa ungueal. Pode existir ainda atrofia da matriz ungueal, queratose subungueal, hiper- pigmentação subungueal e eritroníquia ou melanoníquia. $^{3-4}$

O diagnóstico é baseado na clínica e pode ser complementado pela biópsia das lesões. ${ }^{2}$ A histopatologia é geralmente caracterizada por um infiltrado linfohistocítico, denso, contínuo na junção dermoepidérmica e na derme superior. ${ }^{2}$

O diagnóstico diferencial do líquen cutâneo caracteriza-se por patologias que podem simular as características das lesões de líquen, como os eczemas, o líquen simples crónico, a pitiríase rósea, o prurigo nodular e a psoríase. ${ }^{3}$

Existem alguns fatores de risco descritos na literatura que incluem stress/ansiedade, infeção pelo vírus da hepatite $\mathrm{C}$, doenças autoimunes, doença maligna, dislipidemia e infeções víricas. ${ }^{2} \mathrm{Na}$ literatura é descrita uma associação entre a infeção pelo vírus da hepatite C e o líquen plano, pelo que se considera pertinente fazer o rastreio da hepatite $\mathrm{C}$ nestes doentes. ${ }^{3,5}$

O líquen plano cutâneo pode resolver espontaneamente em um ou dois anos. Estima-se que cerca de 15 a $20 \%$ dos doentes possam ter períodos de remissão e recorrência. ${ }^{6} \mathrm{O}$ foco do tratamento deverá acelerar a resolução e tratar o prurido. ${ }^{7}$

Não existe evidência sobre qual o tratamento mais eficaz para esta doença. ${ }^{6}$

O tratamento tópico com corticoterapia de alta potência pode ser considerado o tratamento de primeira linha para o líquen cutâneo, em especial nas formas menos extensas da doença. ${ }^{3,8}$ Pelo risco de atrofia cutânea estes doentes devem ser avaliados dentro de duas a três semanas após o início do tratamento. ${ }^{8}$ No caso relatado, o paciente iniciou tratamento com butirato de hidrocortisona $1 \mathrm{mg} / \mathrm{g}$ na formulação de creme, na posologia de duas aplicações diárias nas lesões. Este é considerado um corticosteroide de baixa potência, o que poderá explicar o insucesso da terapêutica que foi utilizada inicialmente.

A segunda linha de tratamento consiste na utilização de corticoterapia sistémica, fototerapia ou acitretina oral. Os riscos e benefícios do tratamento devem ser cuidadosamente considerados antes do início da terapêutica. ${ }^{8}$ Uma vez que o paciente apresentava agravamento das lesões após realizar tratamento com corticoterapia tópica e, tinha que aguardar nove meses para a consulta hospitalar de dermatologia, optou-se por 
não seguir a segunda linha de tratamento, nomeadamente a fototerapia, que teria que ser realizada a nível hospitalar, mas seguir a indicação do colega de dermatologia e realizar tratamento com griseofulvina off-label.

Em relação ao tratamento sistémico, o clássico é o uso corticoterapia sistémica. Habitualmente utiliza-se a dexametasona na dose de 30-60mg/dia. ${ }^{3,6,9} \mathrm{O}$ tempo de tratamento descrito na literatura é variável, podendo estar compreendido entre o mínimo de quatro semanas até ao máximo de seis meses. ${ }^{3,6,8-9} \mathrm{~A}$ corticoterapia sistémica por períodos prolongados deve ser ponderada cautelosamente, tendo em conta os efeitos laterais e pouca evidência disponível.

A fototerapia pode ser utilizada tanto com radiação ultravioleta B (UVB), como com radiação ultravioleta A com psoralenos (PUVA). A evidência em relação a esta terapêutica é limitada. ${ }^{8}$ Foram referidos casos de exacerbação da doença após fototerapia. ${ }^{6,9}$ Os principais efeitos laterais são queimaduras, prurido e formação de flictenas e vesículas. ${ }^{8}$

A terapia com acitretina, um retinóide oral, tem pouca evidência e deve estar restrita às formas severas de líquen cutâneo e a médicos com experiência com o fármaco. Este fármaco é teratogénico, pelo que está contraindicado em mulheres em idade fértil., ${ }^{3,8}$

Outros fármacos têm sido utilizados em ensaios clínicos, não tendo ainda aprovação para o tratamento desta patologia, entre os quais: talidomida, griseofulvina, ciclosporina, dapsona, sulfasalazina, metronidazol, azatioprina, itraconazol, micofenolato de mofetilo, fenitoína, ciclofosfamida, interferão e hidroxicloroquina. A eficácia e efeitos laterais são variáveis. ${ }^{6,8-9}$

A griseofulvina mostrou melhorias clinicamente significativas num estudo com 44 doentes, duplamente cego, controlado com placebo em que foi utilizada a dose de 500mg/dia, tendo-se verificado uma melhoria de $91,6 \%$ no grupo de tratamento e uma melhoria de $20,8 \%$ no grupo de controlo. Nos estudos realizados com esta substância não foram reportados efeitos laterais significativos. ${ }^{10}$

Os anti-histamínicos orais podem ser úteis para o controlo do prurido. ${ }^{8}$
O líquen plano é uma doença pouco frequente pelo que a descrição deste caso pretende alertar o médico de família para esta patologia e realçar a importância da comunicação via ALERT® ou via outros instrumentos de eficaz articulação entre os cuidados de saúde primários e secundários. Este caso é um exemplo em que essa articulação de cuidados permitiu um diagnóstico e orientação terapêutica atempada.

\section{REFERÊNCIAS BIBLIOGRÁFICAS}

1. Barreiro D, Santiago LM. Motivos de consulta em medicina geral e familiar no distrito de Coimbra no ano de 2010 [Reasons for clinical encounter in general practice/family medicine in 2010 in Central Portugal]. Rev Port Med Geral Fam. 2013;29(4):236-43. Portuguese

2. Gorouhi F, Davari P, Fazel N. Cutaneous and mucosal lichen planus: a comprehensive review of clinical subtypes, risk factors, diagnosis, and prognosis. Scientific World J. 2014;2014:ID742826.

3. Usatine RP, Tinitigan M. Diagnosis and treatment of lichen planus. Am Fam Physician. 2011;84(1):53-60.

4. Fitzpatrick $T$, Johnson R, Wolff $K$, Suurmond D. Dermatologia: atlas e texto. $5^{\text {a }}$ ed. Rio de Janeiro: McGraw-Hill; 2006. ISBN 9788577260072

5. Shengyuan L, Songpo Y, Wen W, Wenjing T, Haitao Z, Binyou W. Hepatitis $C$ virus and lichen planus: a reciprocal association determined by a meta-analysis. Arch Dermatol. 2009;145(9):1040-7.

6. Asch S, Goldenberg G. Systemic treatment of cutaneous lichen planus: an update. Cutis. 2011;87(3):129-34.

7. Le Cleach L, Chosidow O. Clinical practice: lichen planus. N Engl J Med. 2012;366(8):723-32.

8. Goldstein B, Goldstein A, Mostow E. Lichen planus. UpToDate. 2014 Feb 4. Available from: http://www.uptodate.com/contents/lichen-planus

9. Cribier B, Frances C, Chosidow O. Treatment of lichen planus: an evidence-based medicine analysis of efficacy. Arch Dermatol. 1998;134(12):1521-30.

10. Fazel N. Cutaneous lichen planus: a systematic review of treatments. J Dermatolog Treat. 2015;26(3):280-3.

\section{CONFLITO DE INTERESSES}

Os autores declaram não ter conflitos de interesses.

\section{ENDEREÇO PARA CORRESPONDÊNCIA}

Ana Sofia Nunes Pereira de Sousa e Silva

R. José Augusto Castro, $98-2^{\circ} \mathrm{A}, 4150-002$ Porto

E-mail: sofiasousaesilva@gmail.com

Recebido em 09-06-2015

Aceite para publicação em 30-01-2016

Artigo escrito ao abrigo do novo acordo ortográfico. 


\section{ABSTRACT}

\section{LICHEN PLANUS: A CASE OF SUCCESSFUL TEAMWORK}

Introduction: Skin complaints are a common cause for visits to the primary care physician. This case report describes a patient diagnosed with lichen planus, which is an uncommon health problem. It is a chronic, inflammatory, immune-mediated disease of unknown etiology that affects from 0.2 to $5 \%$ of the population.

Case report: A 60 year-old male patient, previously in good health, saw his family physician in October 2014 complaining of pruritic papules on the hands, trunk, back and feet, which appeared one month before. He also had pain on pressure over his fingernails. Physical examination showed polygonal, flat, well-defined purple papules, grouped and confluent, on the anterior surface of the wrists, chest, back, and the ventral region of the ankle. The patient also presented nail clubbing, erythema on the anterior surface of the hands, periungueal erythema affecting the matrix, thinning, and longitudinal grooves of the nail surface and pain on palpation of the root of the nails. The primary care physician requested dermatology consultation via the online referral service (ALERT ${ }^{\circledR}$ ). The dermatologist suggested the diagnosis of lichen planus. The patient began therapy with a topical corticosteroid, which was not helpful. He subsequently started treatment with griseofulvin, $500 \mathrm{mg}$ per day, which was effective.

Comment: This case report emphasizes the importance of communication between specialists to address the patient's problems, in order to expedite treatment and improve quality of life.

Keywords: Lichen planus; Griseofulvin. 